\title{
Scratchpad Memories for Parallel Applications in Multi-core Architectures *
}

\author{
Francis Birck Moreira, Eduardo Henrique Molina da Cruz, \\ Marco Antonio Zanata Alves, Philippe Olivier Alexandre Navaux \\ Grupo de Processamento Paralelo e Distribuído \\ Instituto de Informática \\ Universidade Federal do Rio Grande do Sul \\ \{fbmoreira, ehmcruz, mazalves, navaux $\}$ inf.ufrgs.br
}

\begin{abstract}
Scratchpad memories are largely used in embedded processors due to their reduced energy consumption and area compared to traditional cache memories. In multi-core architectures, these memories are an interesting solution for the storage of shared data and data which is used intensively. However, these memories present some challenges, such as the need for manual choice of the content. Furthermore, different sizes of scratchpad memories result in the need to modify the source code of the application. In this article, we propose the use of a scratchpad memory in a multi-core architecture which alleviates these disadvantages. We added the scratchpad to an architecture consisting of 4 cores, reducing the size of L2 cache in order to give chip area to the scratchpad memory. We evaluated our proposed design by executing the NAS Parallel Benchmark (NPB) applications in a simulator. We improved performance by up to $45 \%$ compared to the base architecture, reducing cache invalidations by up to $85 \%$.
\end{abstract}

\section{Introdução}

Arquiteturas multi-core são o foco do estado da arte no desenvolvimento de novas arquiteturas de processadores. Nestas arquiteturas, há um aumento substancial na quantidade de transações de dados entre a memória primária e o processador devido ao crescente aumento no número de núcleos de processamento. Assim, a crescente diferença de desempenho entre a memória principal e o processador ressalta o sub-sistema de memória como um dos principais gargalos das novas arquiteturas multi-core [1]. Logo,

\footnotetext{
*Trabalho parcialmente apoiado pelo CNPq e CAPES.
}

a utilização de memórias cache vem sendo empregada de maneira a mascarar a latência da memória primária, valendo-se de vários níveis de hierarquia e mecanismos avançados, como pipelining e prefetching.

Entretanto, dados compartilhados por diferentes memórias cache podem acarretar perda de desempenho [4]. Um dos principais motivos para isto é a replicação descontrolada de dados em diferentes caches, o que diminui o espaço útil da memória cache. Os protocolos de coerência de memória cache, necessários para validação de acessos concorrentes, também impõem sobrecargas em sistemas com múltiplas caches. Isto ocorre porque escritas em linhas de cache geram invalidações em outras memórias caches que compartilham a linha sendo escrita [3].

A memória scratchpad (Scratchpad Memory - SPM) [6] é uma memória especializada visível ao processador e o sistema operacional. Nas arquiteturas multi-core, tal memória pode ser compartilhada entre os núcleos de processamento, provendo acesso eficiente a dados compartilhados. O scratchpad representa uma extensão ao espaço de armazenamento presente nas arquiteturas multi-core, sendo que o controle dos dados que o populam é feito via software.

Pesquisas com o scratchpad indicam ganhos principalmente em termos de área e energia [13]. Isto deve-se ao fato de implementações de memórias scratchpad não utilizarem tags e comparadores, que são necessários em memórias cache tradicionais. Além disso, uma memória scratchpad compartilhada não requer protocolos de coerência, já que todos os núcleos operam sobre os mesmos dados. Tais características tornam o uso do scratchpad interessante em sistemas embarcados, onde o consumo de potência e área do chip são muitas vezes mais importantes que o desempenho.

Neste contexto, torna-se interessante avaliar o uso da memória scratchpad em um sistema multi-core de propósito geral. Aliada à memória cache tradicional, o scratchpad representa uma solução eficiente para reduzir o consumo 
de energia da hierarquia de memória como um todo. Ele provê um espaço de armazenamento eficiente para estruturas de dados determinadas pelo programador, compilador ou sistema operacional, o que acarreta ganho de desempenho dada uma escolha de dados inteligente, priorizando dados frequentemente acessados.

O objetivo deste trabalho é propor e avaliar o desempenho de sistemas multi-core com o uso de memórias scratchpad. Para isto, desenvolveu-se uma simulação de memória scratchpad em um ambiente multi-core formado de 4 núcleos de processamento. O scratchpad foi implementado no simulador Simics [9], integrando-se como um componente da hierarquia de memória, modelado de maneira inovadora ao considerar um controle de páginas no Translation Look-Aside Buffer(TLB). As aplicações do benchmark NAS Parallel Benchmarks (NPB) [7] serviram de base para os experimentos, sendo que seus dados compartilhados e frequentemente acessados pelos múltiplos núcleos foram armazenados no scratchpad. A escolha de dados das aplicações a serem alocados no scratchpad foi feita com o auxilio da ferramenta VTune [12] da Intel, a qual pode ser utilizada em uma vasta gama de aplicações paralelas, assim auxiliando o programador na escolha dos dados mais importantes a serem mapeados no scratchpad.

Este artigo está organizado da seguinte maneira. A seção 2 apresenta os trabalhos relacionados a área. Na Seção 3, é descrita uma modelagem para o uso da memória scratchpad em uma arquitetura multi-core. A Seção 4 explica a implementação da arquitetura no simulador. Os detalhes da carga de trabalho utilizada são mostrados na Seção 5. A Seção 6 traz a análise sobre os resultados obtidos nos experimentos. Finalmente, a Seção 7 traça as conclusões do artigo.

\section{Trabalhos Relacionados}

No trabalho de Banakar et al. [2], são apresentados comparativos entre utilização da memória scratchpad e a memória cache para sistemas embarcados. Os quesitos mais importantes apontados no estudo foram área e energia, onde demonstrou-se superioridade relevante da memória scratchpad. Entretanto, a programação dos aplicativos na arquitetura apresentada precisa ser feita em baixo nível, onde o programador necessita gerenciar diretamente o conteúdo da memória scratchpad.

O artigo apresentado por Poletti et al.[5] investiga modos eficazes de implementar a arquitetura de memória scratchpad em um ambiente embarcado multi-core sem memória cache. Neste contexto, foi avaliado o uso de um mecanismo de Direct Memory Access, o qual permite alocação eficiente da memória scratchpad em tempo de execução. Além disso, foi descrito também uma interface para o uso da memória scratchpad para ajudar na programação envol- vendo o mesmo. Porém, neste trabalho, o endereçamento da memória depende do tamanho da memória scratchpad e é feito de modo direto, implicando em dificuldade para usar funções e bibliotecas pré-existentes.

$\mathrm{O}$ uso de uma memória scratchpad em um ambiente multi-core também foi explorado por Yanamandra et al. [14], utilizando uma memória scratchpad para cálculos envolvendo matrizes esparsas. $\mathrm{O}$ artigo demonstra a comparação de eficiência com dois experimentos base. No primeiro, parte da memória cache $\mathrm{L} 1$ é removida para dar lugar a uma memória scratchpad; no segundo experimento, a mesma estratégia é adotada na memória cache L2. Os resultados, em termos de desempenho e energia, se mostram favoráveis para a adoção da memória scratchpad no segundo nível da hierarquia de memória. Na descrição do método de simulação da memória scratchpad, é citado o uso do simulador Simics, as latências e as modificações de tamanhos nas caches. Entretanto, não são apresentados detalhes sobre como a memória foi simulada nem sobre como eram feitos os acessos ao scratchpad.

Nguyen et al.[11] demonstra uma técnica para resolução do problema de retrocompatibilidade ao criar um instalador de software customizado, o qual decide a alocação da memória scratchpad exatamente antes da primeira execução do programa. Determinado o tamanho da memória scratchpad, o instalador modifica o executável de acordo com a alocação escolhida. Esta técnica foi aplicada em sistemas embarcados, no qual o uso de um instalador é pertinente, já que atualizações de softwares embarcados são incomuns.

Comparando os trabalhos correlatos, pode-se ressaltar que este estudo considera um conjunto maior de aplicações paralelas, onde utiliza-se uma memória scratchpad compartilhada entre diversos núcleos, a fim de oferecer espaço de memória com acesso eficiente. Assim, reduz-se o tempo de comunicação entre as diversas threads das aplicações paralelas, bem como o tempo de acesso às estruturas mais utilizadas pelas aplicações.

\section{Proposta de uma memória scratchpad em arquiteturas multi-core}

A memória scratchpad foi modelada para oferecer uma memória rápida e paralela à hierarquia de memória cache. Para isso, antes de enviar uma transação para a hierarquia de memória cache, todos os endereços requisitados devem ser verificados para saber se o mesmo encontra-se no scratchpad. Caso o endereço esteja no scratchpad, o mesmo é responsável por tratar a transação. Caso contrário, a transação é repassada para a hierarquia de memória cache. Para a manipulação de quais estruturas de dados estão presentes na memória scratchpad, são usadas chamadas de sistema especificando quais faixa de endereços estão armazenados 
nesta memória. Dessa forma, a análise de endereços presentes no scratchpad se resume em uma comparação de faixas de endereços.

Deste modo, utiliza-se a memória scratchpad como um armazenamento específico para estruturas de dados compartilhadas que são frequentemente usadas. Objetiva-se ganho de desempenho ao fazer uma seleção astuta dos dados mais usados, já que haverá diminuição de dados replicados e, por consequência, menor sobrecarga pelos protocolos de coerência de cache. Na manipulação do conteúdo presente na memória scratchpad por parte dos programas, os endereços são acessados como se fossem endereços normais, pois estes são traduzidos para o espaço da memória scratchpad automaticamente pelo hardware.

Assim é possível usar uma memória scratchpad que se adapta às necessidades de uma arquitetura multi-core genérica. Há retrocompatibilidade ao se mapear o espaço de endereçamento da memória scratchpad em faixas de endereçamento da memória primária. Como a tradução é implementada em hardware, poucas alterações são necessárias nos códigos das aplicações.

Com este modelo, em uma memória scratchpad compartilhada por múltiplos núcleos, verificamos a necessidade de especificarmos um mecanismo para gerenciar a chegada de múltiplas requisições. Para isto, foi utilizado um modelo simples com $N$ portas paralelas, atrasando requisições caso nenhuma porta esteja disponível. Desta forma, foi possível experimentar diferentes números de portas (1-Porta, 2Portas e 4-Portas de acesso), de modo a verificar o problema de contenção de acessos à memória scratchpad, e assim determinar um número de portas adequado para o sistema avaliado.

\section{Simulação e Parâmetros}

O simulador Simics foi empregado para simular um sistema multi-core com 4 núcleos de processamento e uma organização de hierarquia de memória semelhante à encontrada na arquitetura Harpertown, conforme ilustrado na Figura 1. Utilizando este sistema como base, duas arquiteturas foram simuladas: a primeira arquitetura base sem a memória scratchpad, (Figura 1(a)) e a segunda arquitetura com a memória scratchpad (Figura 1(b)). Os núcleos simulados no Simics são baseados em processadores UltraSPARC com o conjunto de instruções V9. Para uma comparação justa, foi reduzida a capacidade das memórias cache L2 no ambiente que utilizou a memória scratchpad. A Tabela 1 sumariza os parâmetros da hierarquia de memória, ressaltando que os parâmetros de latência das memórias foram gerados utilizando a ferramenta CACTI [10], considerando núcleos de processamento UltraSPARC com frequência de $1 \mathrm{GHz}$.

A decisão de projeto que leva a uma memória scratch-

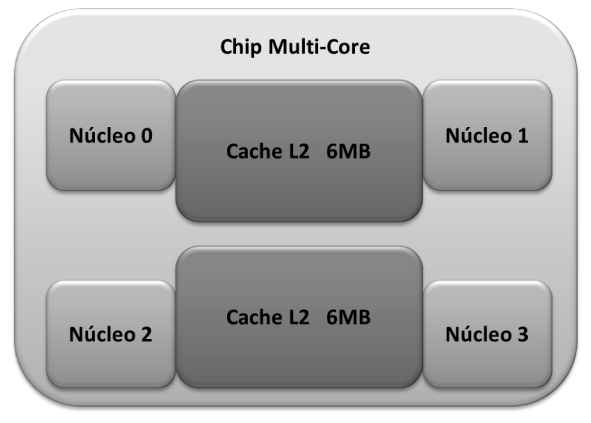

(a) Arquitetura Base.

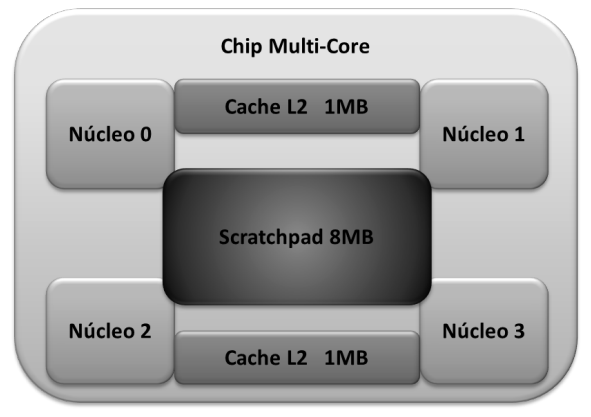

(b) Arquitetura com SPM.

\section{Figura 1. llustração das arquiteturas mode- ladas, 1(a) com apenas memórias cache e 1(b) com memórias cache e Scratchpad.}

pad de grande capacidade $(8 \mathrm{MB})$ se deve à possibilidade de uso desta por vários programas ou threads, sendo que, em sistemas de propósito geral de alto desempenho, diversas aplicações exigem grandes estruturas de dados. Do ponto de vista de implementação física, a diferença de latência e energia entre memórias cache e scratchpad em memórias pequenas (aproximadamente $16 \mathrm{~KB}$ ) é próxima de zero, sendo que essa diferença se torna evidente conforme aumenta-se o tamanho dessas estruturas, conforme verificado no CACTI. Portanto, quanto maior o tamanho das memórias cache $\mathrm{e}$ scratchpad, maior a diferença na latência do acesso e no consumo de energia entre elas.

\section{Carga de Trabalho}

As aplicações usadas nos experimentos constituem o Numerical Aerodynamic Simulation Parallel Benchmark (NAS-NPB), versão 3.3.1, paralelizada com OpenMP. Este benchmark é formado por aplicações relacionadas a métodos numéricos de simulações aerodinâmicas para computação científica. Tais aplicações foram projetadas para comparar o desempenho de computadores paralelos, sendo formadas por kernels de simulação de dinâmica de fluídos computacionais (CFD - Computational Fluid Dy- 


\begin{tabular}{c|c|c|c|c|c|c|c|c|c|c}
\hline \hline \multirow{2}{*}{ Configuração } & \multicolumn{3}{|c|}{ Memória Cache L1 } & \multicolumn{3}{c|}{ Memória Cache L2 } & \multicolumn{3}{c}{ Memória Scratchpad } & \multicolumn{2}{c|}{ Memória Principal } \\
\cline { 2 - 11 } & $\begin{array}{c}\text { Tamanho } \\
\text { da Cache }\end{array}$ & $\begin{array}{c}\text { Conjunto } \\
\text { Assoc. }\end{array}$ & $\begin{array}{c}\text { Latência } \\
\text { (Ciclos) }\end{array}$ & $\begin{array}{c}\text { Tamanho } \\
\text { da Cache }\end{array}$ & $\begin{array}{c}\text { Conjunto } \\
\text { Assoc. }\end{array}$ & $\begin{array}{c}\text { Latência } \\
\text { (Ciclos) }\end{array}$ & $\begin{array}{c}\text { Tamanho } \\
\text { do SPM }\end{array}$ & $\begin{array}{c}\text { Latência } \\
\text { (Ciclos) }\end{array}$ & $\begin{array}{c}\text { Tamanho } \\
\text { da RAM }\end{array}$ & $\begin{array}{c}\text { Latência } \\
\text { (Ciclos) }\end{array}$ \\
\hline \hline Cache normal & $32+32 \mathrm{~KB}$ & 8 Vias & 3 & $6+6 \mathrm{MB}$ & 24 Vias & 15 & - & - & $1 \mathrm{~GB}$ & 78 \\
\hline Cache + SPM & $32+32 \mathrm{~KB}$ & 8 Vias & 3 & $1+1 \mathrm{MB}$ & 16 Vias & 8 & $8 \mathrm{MB}$ & 12 & $1 \mathrm{~GB}$ & 78 \\
\hline Cache 1MB & $32+32 \mathrm{~KB}$ & 8 Vias & 3 & $1+1 \mathrm{MB}$ & 16 Vias & 8 & $8 \mathrm{MB}$ & 12 & $1 \mathrm{~GB}$ & 78 \\
\hline $\begin{array}{c}\text { Cache 1MB, 15 } \\
\text { ciclos }+ \text { SPM }\end{array}$ & $32+32 \mathrm{~KB}$ & 8 Vias & 3 & $1+1 \mathrm{MB}$ & 16 Vias & 15 & $8 \mathrm{MB}$ & 12 & $1 \mathrm{~GB}$ & 78 \\
\hline
\end{tabular}

Tabela 1. Parâmetros utilizados na simulação dos experimentos.

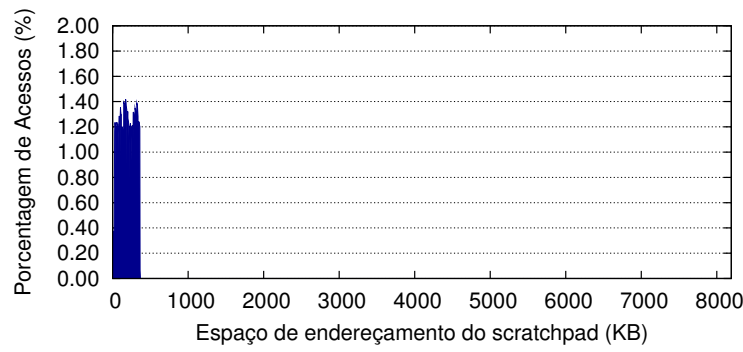

(a) $\mathrm{BT}$

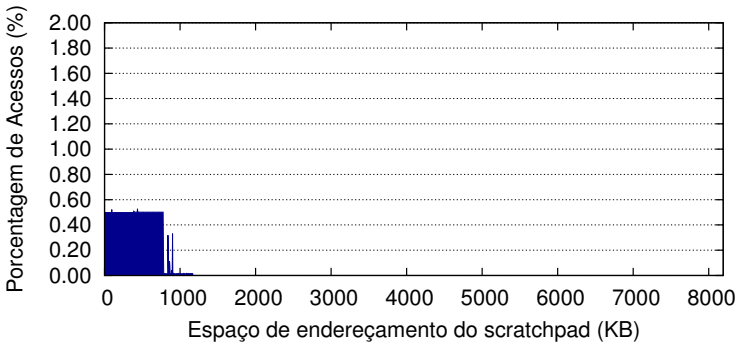

(b) $\mathrm{CG}$

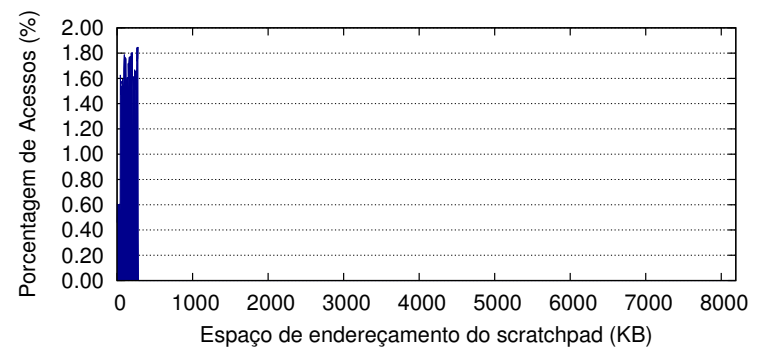

(c) $\mathrm{LU}$

\section{Figura 2. Histograma de uso das regiões da memória scratchpad para as aplicações que utilizaram menos que 4 MB da memória scratchpad.}

namics) derivados de importantes aplicações das classes aerofísicas, de maneira que as simulações reproduzem grande parte dos movimentos de dados e computações encontrados em códigos de dinâmica de fluídos completos [7].

As seguintes aplicações fazem parte deste estudo: BT (Block Tridiagonal), CG (Conjugate Gradient), MG (Multigrid), EP (Embarassingly Parallel), SP (Scalar Pentadiagonal), LU (Lower and Upper Triangular System), IS (Integer Sort), FT (Fast Fourier Transform), UA (Unstructured Adaptive). Todas essas aplicações executam operações de ponto-flutuante de precisão dupla (64 bits) e são programadas utilizando Fortran-90 (BT, CG, EP, FT, LU, MG, SP, UA), com exceção da aplicação IS, programada em C, efetuando operações sobre inteiros.

As aplicações BT, SP e LU possuem seu paralelismo através da divisão de domínio espacial, com compartilhamento de dados nas bordas de cada subdomínio, apresentando acessos de forma linear aos dados. As aplicações CG e UA apresentam características de acesso randômico aos dados. A aplicação MG trabalha sobre dados contíguos, variando o padrão de acesso a cada etapa de computação, indo de acessos desalinhados até acessos lineares conforme a grade é refinada. Assim, esta aplicação pode ser considerada um mistro entre as aplicações de acesso regular (BT, LU e SP) e as de acesso irregular (CG e UA).

A aplicação EP é completamente paralela e possui poucos acessos à memória. O desempenho dessa aplicação pode ser utilizado como referência de desempenho computacional de pico de uma determinada máquina. A aplicação IS apresenta comportamento contrário a aplicação EP, já que executa muitos acessos a dados compartilhados, além dos acessos lineares aos dados armazenados. Por fim, a aplicação FT apresenta etapas de acesso linear com etapas de acesso compartilhado entre as diversas threads.

O NAS-NPB conta com vários tamanhos de entrada para os problemas, S, W, A, B, C (partindo do menor para o maior), sendo que para este trabalho foi utilizado o tamanho W. Este tamanho é o mais indicado para simulações, já que 


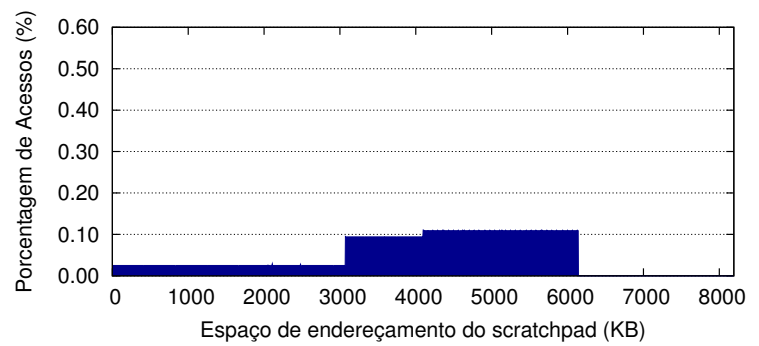

(a) FT

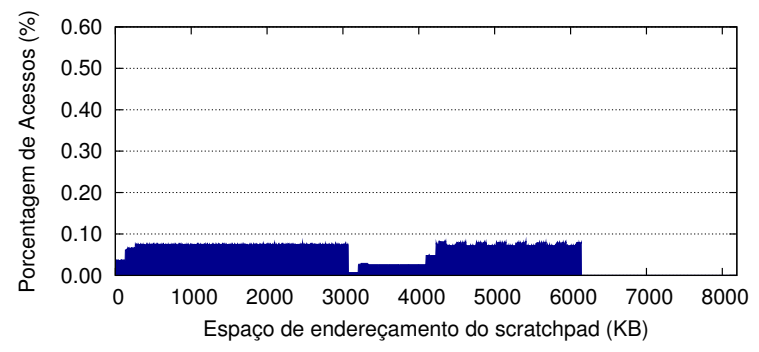

(c) $\mathrm{MG}$

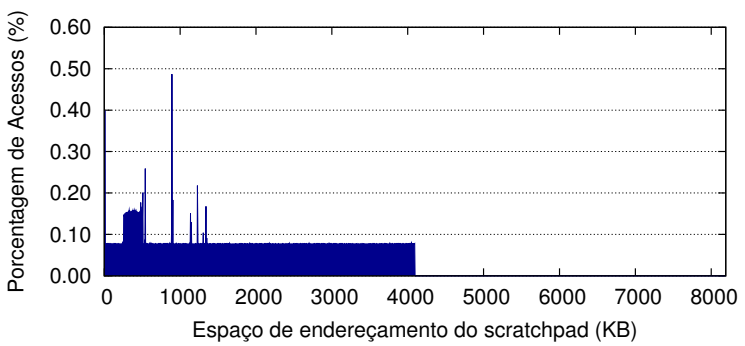

(b) IS

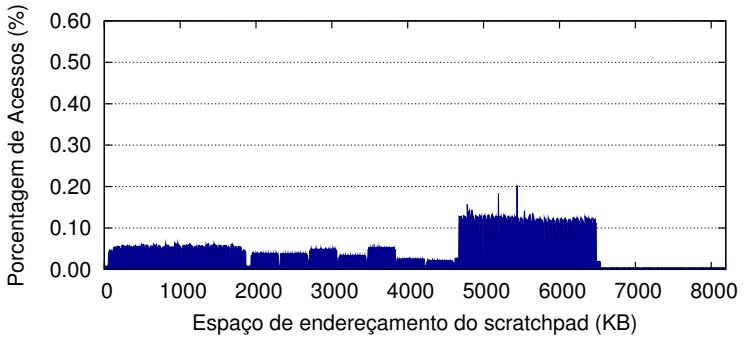

(d) $\mathrm{SP}$

\section{Figura 3. Histograma de uso das regiões da memória scratchpad para as aplicações que utilizaram mais que 4 MB da memória scratchpad.}

é classificado como um problema de tamanho real e, mesmo assim, possui um tempo viável de simulação.

\section{Resultados experimentais}

Para observar e comparar as execuções com a memória scratchpad e com a memória cache foram feitas algumas medidas. Mediu-se tempo de execução com objetivo de medir o desempenho obtido. Foram obtidas também medidas de uso da memória scratchpad para observar o quão efetivamente usada esta foi, podendo-se observar o padrão de acessos nas estruturas alocadas. O número de mensagens de coerência entre as memórias cache L2 foi analisado para visualizar o quanto a memória scratchpad ajuda a aliviar a sobrecarga destes, melhorando a performance das caches. E, por final, é feita uma comparação entre a memória cache L2 de tamanho reduzido, com latência de 8 ciclos, sem o uso do scratchpad, e memória cache L2 com latência de 15 ciclos, com o uso da memória scratchpad. O objetivo é comprovar que o uso da memória scratchpad é o responsável pela melhoria de performance, e não apenas a redução da latência da memória cache L2.

Para a alocação das estruturas de dados nos programas, foi necessário modificar o código dos benchmarks usados, de forma a fazer as chamadas ao Simics descrita nas seções 3 e 4 e armazenar as estruturas desejadas na memória scratchpad. Para selecionar tais estruturas, foi usado o programa Vtune [12] da Intel, o qual utiliza hardware performance counters presentes nos processadores da Intel para fazer profiling de aplicações. Com isto, é possível identificar quais estruturas de dados demandam mais tempo de execução, sendo estas as estruturas que foram armazenadas na memória scratchpad.

Todas as aplicações foram alteradas de maneira a se realizar uma alocação estática da memória scratchpad logo no início da execução. Foram obtidos resultados de todas aplicações do NPB, exceto da aplicação DC (Data Cube Operator), a qual não executaria em tempo viável em ambientes de simulação. São fornecidas as diferenças de tempo de execução para cada benchmark e um histograma apresentando a frequência de acesso a cada parcela de endereços dentro da memória scratchpad. Isto permite analisar o uso da memória e as diferenças entre as regiões de cada estrutura de dados armazenada.

Observa-se, no benchmark BT (Block Tridiagonal), o uso de uma parcela pequena da memória scratchpad, conforme a Figura 2(a), usando aproximadamente $368 \mathrm{~KB}$ da memória scratchpad. Ainda assim, os resultados apresentam ganhos de $21,75 \%$, conforme a Figura 4, devido à alta concentração de acessos nesta parcela de endereços. Observando a estrutura do programa, é possível assumir que toda a estrutura principal foi alocada (para o tamanho W, uma matriz tridimensional de $33 \times 33 \times 33$ ) na memória scratchpad. Porém, tal estrutura cabe nas memórias cache L2 de $1 \mathrm{MB}$, e apenas as reduções de tamanho e latência da memória cache L2 provam-se necessárias para ganho de desempenho, como pode ser observado na Figura 5. Há ganho de desempenho ao se utilizar a memória scratchpad, 


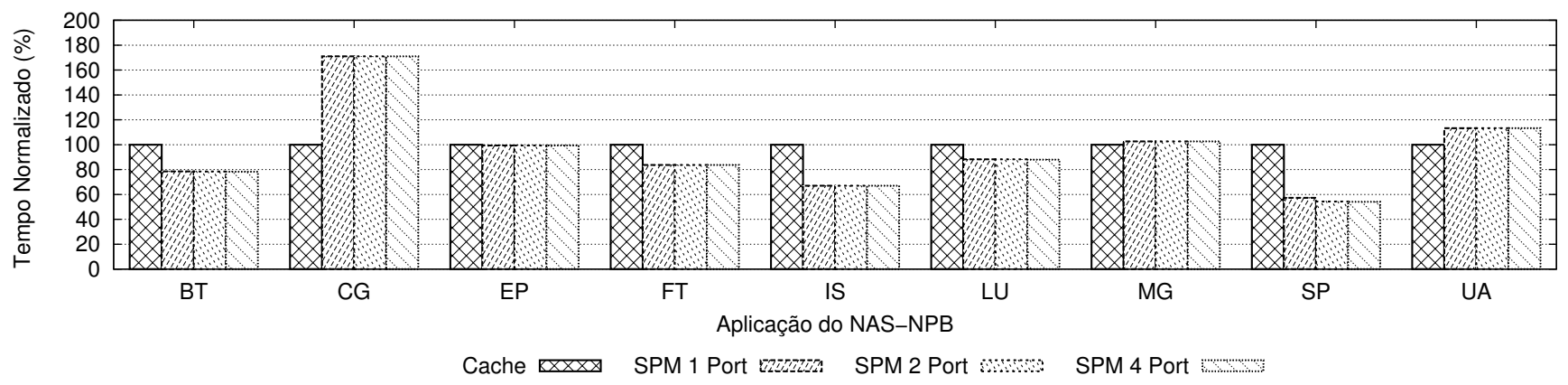

Figura 4. Tempo de execução dos benchmarks para configurações com memória cache e memória scratchpad com 1, 2 e 4 portas.

pois há redução no número de invalidaçõese mensagens de modificação de estado entre as memórias cache L2, conforme visto na Figura 6.

O padrão randômico de acessos à memória presente no benchmark CG (Conjugate Gradient) impõe uma maior sobrecarga no mecanismo de caching de dados. Como a cache $\mathrm{L} 2$ fora reduzida, houve perda de desempenho, pois a memória scratchpad não fora utilizada o bastante para compensar a memória cache L2 menor. Os tempos de execução do benchmark apresentam perda de $71,03 \%$, na Figura 4 . A frequência do uso de dados conforme a faixa de endereços alocada se encontra na Figura 2(b). Para o tamanho de entrada $\mathrm{W}$, o benchmark utiliza uma matriz esparsa positiva de 7.000 posições e, como pode observar-se na Figura 5, uma arquitetura usando uma memória cache de tamanho e latência reduzidos também perde desempenho. Adicionalmente, a Figura 6 mostra que o número de mensagens de coerência entre as memórias cache L2 aumentou, comprovando que a faixa de endereços selecionada para a memória scratchpad não foi a mais frequentemente acessada, e que as memórias cache L2 com 1MB não tem condições de prover dados eficientemente.

O benchmark EP (Embarassingly Parallel) usa o método polar Marsaglia para gerar pares de números randômicos, o que necessita de muito pouca memória. Na Figura 4, é demonstrado que não existe diferença significante entre os tempos de execução. Foi armazenado no scratchpad uma faixa de endereços considerada relevante, mas que possui tamanho insignificante perante o tamanho total do scratchpad. Os dados alocados na memória scratchpad são pouco acessados, gerando nenhum ganho de desempenho. Como observado na Figura 5, o uso da memória scratchpad foi insignificante, pois o tempo de execução com uma memória cache $\mathrm{L} 2$ de $1 \mathrm{MB}$ e 15 ciclos de latência apresentou perda de desempenho, mesmo que o número de mensagens de coerência entre as memórias cache L2 tenha sido reduzido, conforme a Figura 6.

O benchmark FT (Fast Fourier Transform) realiza a transformada rápida de Fourier em uma grande estrutura matricial tri-dimensional, com dimensões de 128,128 e 32 . Os acessos são bem distribuídos e regulares, conforme a Figura 3(a). A alocação de grande parte da estrutura na memória scratchpad resulta em ganho de desempenho de 18,30\%, como observado na Figura 4. Porém, conforme observado na Figura 5, a estrutura principal mais acessada cabe na memória cache $\mathrm{L} 2$ de $1 \mathrm{MB}$, pois tal ambiente de simulação resulta no mesmo ganho de desempenho em relação ao ambiente usando uma memória scratchpad. A Figura 6 demonstra que houve uma grande queda no número de mensagens de coerência entre as memórias cache L2, embora o número de mensagens para este benchmark seja pequeno, fazendo com que a adição da memória scratchpad torne-se de pouca ou nenhuma relevância neste caso.

O benchmark IS (Integer Sort) realiza a classificação de um vetor de inteiros de 1.048.576 posições, e apresenta acessos randômicos à memória tal qual o benchmark CG. Porém, como foi possível popular mais dados no scratchpad em relação ao CG, armazenando a estrutura inteira dentro da memória scratchpad, ganhos de desempenho mais expressivos foram obtidos, de 33,12\% conforme a Figura 4. A frequência de acesso aos dados é inconstante na estrutura alocada, como pode ser observado na Figura 3(b). A memória scratchpad desempenha um papel essencial em tal benchmark, como pode ser observado na Figura 5, pois a estrutura principal não cabe nas memórias cache. A Figura 6 demonstra a queda nas mensagens de coerência, pois com a memória scratchpad não é necessário ficar substituindo partes da estrutura na memória cache L2.

O benchmark LU (Lower and Upper Triangular System) consiste de um algoritmo de resolução de sistemas tal qual o BT, e resolve um sistema de equações não lineares de uma matriz $33 \times 33 \times 33$. Ele apresentou ganho de desempenho de $12,15 \%$, conforme a Figura 4, mesmo usando-se apenas uma parcela pequena da memória scratchpad, como pode ser observado na Figura 2(c). Igualmente ao BT, a 


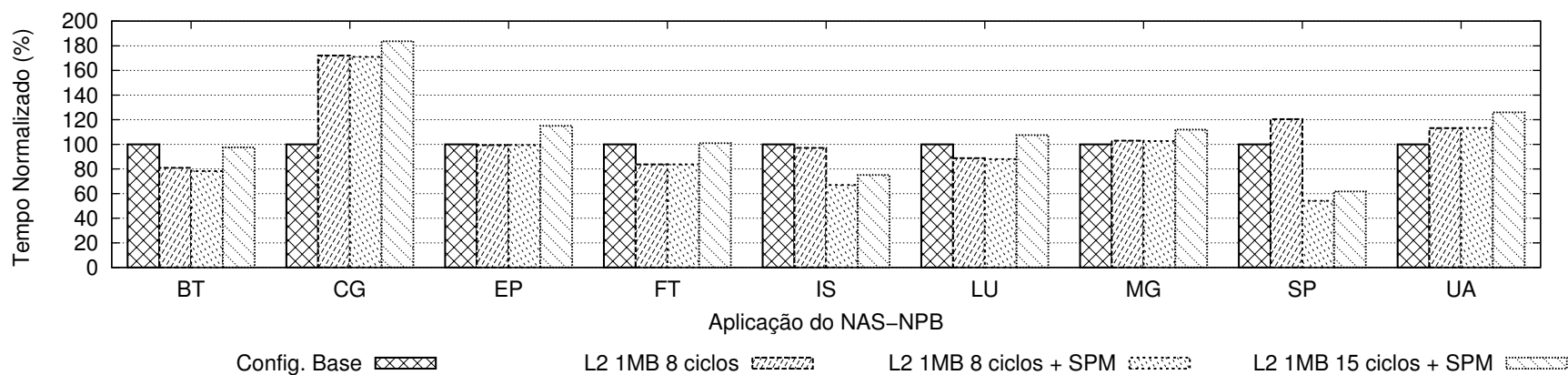

Figura 5. Comparação do scratchpad com cache normal 6MB, cache de $1 \mathrm{MB}$ e latência de 8 ciclos, cache de $1 \mathrm{MB}$ e latência de 8 ciclos com scratchpad e cache de 1MB e latência de 15 ciclos com scratchpad

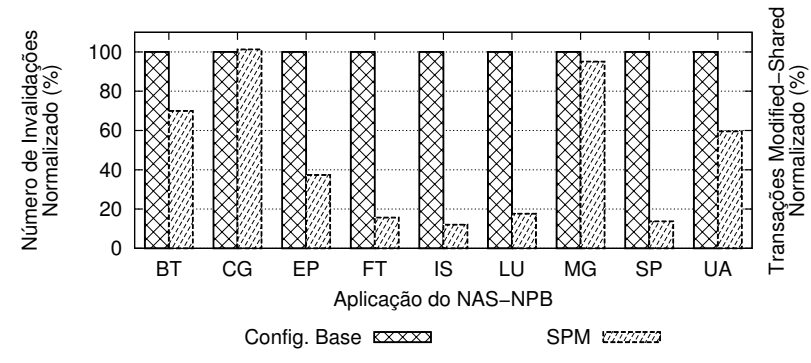

(a) Invalidações

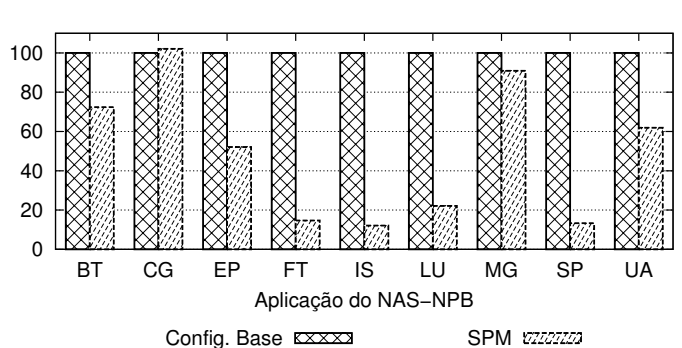

(b) Modified to Shared

\section{Figura 6. Quantidade de invalidações e mudanças de estado de modified para shared detectadas no protocolo MESI.}

estrutura inteira cabe nas memórias cache $\mathrm{L} 2$ de $1 \mathrm{MB}, \mathrm{o}$ que resulta em ganho de performance semelhante sem o uso de memória scratchpad, conforme a Figura 5. A vantagem obtida com a memória scratchpad é a redução de mensagens de coerência, observada na Figura 6, a qual gera a diferença de desempenho entre memórias cache L2 sem uso de scratchpad e com uso de scratchpad.

O benchmark MG (Multigrid) resolve uma equação discreta de Poisson tridimensional com dimensões de 128x128x128, com acessos iniciais esparsos onde a distância entre os acessos reduz até torna-los regulares, facilitando o uso de grande parcela da memória scratchpad. Pode-se observar na Figura 3(c) que os acessos distribuemse uniformemente em duas grandes faixas de dados na memória scratchpad. Entretanto, não obtêm-se ganho de desempenho, pois o scratchpad não tem capacidade para armazenar todos os dados do MG, que aparenta possuir estruturas mais usadas que a matriz principal. Desta forma, a diminuição da memória cache de nível 2 impõe uma perda de desempenho superior aos ganho obtidos com o scratchpad, como observado na Figura 5. Adicionalmente, pode-se observar pela pequena redução do número de mensagens de coerência na Figura 6, que a seleção das estruturas de dados não foi ideal.
Tal qual os benchmarks BT e LU, o benchmark SP (Scalar Pentadiagonal) resolve de forma paralela um conjunto de sistemas não lineares de uma matriz de dimensões $33 \times 33 \times 33$, com acessos regulares. Entretanto, nessa aplicação, a maior parcela do espaço de endereçamento do scratchpad foi aproveitado (Figura 3(d)), assim, ganhos mais expressivos, de $45 \%$ foram obtidos. Diferentemente dos benchmarks BT e LU, o SP aloca três grandes vetores utilizados para manipulação dos dados, os quais foram armazenados na memória scratchpad, tornando a memória scratchpad essencial ao ganho de desempenho, conforme mostrado na Figura 5. Neste benchmark, o número de mensagens de coerência é reduzido em aproximadamente $90 \%$, como visto na Figura 6. Devido ao número intensivo de mensagens trocadas, é notado que a memória cache L2 de $1 \mathrm{MB}$, sem o uso de scratchpad, sofre perda de desempenho notável tanto por falta de espaço de armazenamento quanto por mensagens de coerência.

O benchmark UA (Unstructured Adaptive) realiza cálculos de transferência de calor e, assim como os benchmarks CG e IS, possui acessos a memória de forma randômica. Porém, este algoritmo faz alocação dinâmica da memória, sendo inapropriado para o método de alocação estática utilizado no presente trabalho. Obteve-se perda de 
desempenho de 13,29\%, conforme observado na Figura 4, pois alocou-se apenas $4 \mathrm{~KB}$ de memória. A Figura 5 comprova a necessidade de memória cache L2, e, mesmo a redução de mensagens de coerência, vista na Figura 6, não é suficiente para compensar a perda de desempenho. No caso da aplicação UA, os resultados negativos eram esperados, pois além da falta de estruturas a serem alocadas no scratchpad, esta aplicação foi desenvolvida de maneira a se limitar ganhos através da manipulação de conteúdo de memória por compiladores e hardware especializado [7].

\section{Conclusões}

Este trabalho avaliou o potencial do uso de memórias scratchpad em arquiteturas multi-core. Observa-se ganho de desempenho proporcional ao uso efetivo da memória scratchpad. Isto deve-se ao fato de que consegue-se acessar dados compartilhados de forma mais eficientemente do que em memórias cache, já que as últimas ocasionam replicações e sofrem com invalidações por protocolos de coerência. O scratchpad também apresenta menor latência que a memória cache tradicional, pois não necessita de comparação de tags.

Conforme os resultados dos vários benchmarks, nota-se que a memória scratchpad se comporta como uma solução interessante para a grande maioria destes. Ganhos de até $45 \%$ foram obtidos, com uma média de 7,47\%. Aplicações com pouco uso do scratchpad, como o EP e LU, não obtiveram ganho de desempenho, como esperado. Neste trabalho, uma ferramenta de profiling de aplicações foi utilizada para facilitar a tomada de decisões sobre quais estruturas de dados seriam armazenadas no scratchpad simulado. Foi notável a facilidade de modificação do código, pois a hierarquia de memória modelada no artigo considera a facilidade de programação como um fator importante para o sucesso de uma melhoria em uma máquina off-the-shelf, devido ao uso mais variado de tal tipo de máquina.

Para obtenção da melhor performance com os benchmarks, estamos analisando a possibilidade de se implementar uma mudança no algoritmo do compilador demonstrado em [8], para utilizar a memória scratchpad da maneira mais efetiva possível sem aumentar o esforço do programador. Também é possível a aplicação das ferramentas de profiling descritas em [3], que facilitariam a alocação para todos benchmarks, em especial o benchmark CG. Outro foco de futura pesquisa seriam métodos de definição do conteúdo do scratchpad apropriados para alocações dinâmicas de memória, como presente na aplicação UA.

\section{Referências}

[1] M. A. Z. Alves, H. C. Freitas, and P. O. A. Navaux. Investigation of shared 12 cache on many-core processors. In
Proceedings Workshop on Many-Core, pages 21-30, Berlin, 2009. VDE Verlag.

[2] R. Banakar, S. Steinke, B. Lee, M. Balakrishnan, and P. Marwedel. Scratchpad memory: design alternative for cache onchip memory in embedded systems. In Proceedings of the tenth international symposium on Hardware/software codesign, pages 73-78, New York, NY, USA, 2002. ACM.

[3] E. Cruz, M. Alves, and P. Navaux. Process mapping based on memory access traces. In 11th Symposium on Computing Systems, WSCAD-SCC, pages 72-79, 2010.

[4] E. Cruz, C. P. Ribeiro, M. Alves, A. S. Carissimi, J. Mehaut, and P. Navaux. Process mapping based on memory access traces. In 13th Workshop on Advances in Parallel and Distributed Computational Models, APDCM / IPDPS, Los Alamitos, CA, USA, 2011. IEEE Computer Society.

[5] P. Francesco, P. Marchal, D. Atienza, L. Benini, F. Catthoor, and J. M. Mendias. An integrated hardware/software approach for run-time scratchpad management. In Proceedings of the 41st annual Design Automation Conference, DAC, pages 238-243, New York, NY, USA, 2004. ACM.

[6] B. Jacob, S. Ng, and D. Wang. Memory systems: cache, DRAM, disk. Morgan Kaufmann Publishers Inc., 2007.

[7] H. Jin, M. Frumkin, and J. Yan. The OpenMP implementation of NAS parallel benchmarks and its performance. NASA Ames Research Center, Technical Report NAS-99-011, 1999.

[8] L. Li, L. Gao, and J. Xue. Memory coloring: a compiler approach for scratchpad memory management. In Parallel Architectures and Compilation Techniques, 2005. PACT 2005. 14th International Conference on, pages 329-338, 2005.

[9] P. S. Magnusson, M. Christensson, J. Eskilson, D. Forsgren, G. Hallberg, J. Hogberg, F. Larsson, A. Moestedt, and B. Werner. Simics: A full system simulation platform. Computer, 35:50-58, 2002.

[10] N. Muralimanohar, R. Balasubramonian, and N. Jouppi. Optimizing nuca organizations and wiring alternatives for large caches with cacti 6.0. In Proceedings of the 40th Annual IEEE/ACM International Symposium on Microarchitecture, pages 3-14, Los Alamitos, CA, USA, 2007. IEEE Computer Society.

[11] N. Nguyen, A. Dominguez, and R. Barua. Memory allocation for embedded systems with a compile-time-unknown scratch-pad size. ACM Trans. Embed. Comput. Syst., 8:2132, April 2009.

[12] J. Reinders. VTune performance analyzer essentials: measurement and tuning techniques for software developers. Intel Press, 2005.

[13] V. Suhendra, A. Roychoudhury, and T. Mitra. Scratchpad allocation for concurrent embedded software. ACM Transactions on Programming Languages and Systems, 32:13-47, April 2010.

[14] A. Yanamandra, B. Cover, P. Raghavan, M. Irwin, and M. Kandemir. Evaluating the role of scratchpad memories in chip multiprocessors for sparse matrix computations. In IEEE International Symposium on Parallel and Distributed Processing., IPDPS, pages 1-10, 2008. 